\title{
Opportunity of Accelerating User eXperience (UX) Technologies on Embedded Systems
}

\author{
Hyun-Min Choi and Seung Eun Lee* \\ Dept. of Electronic \& Information Technology \\ Seoul National University of Science and Technology, Seoul, Korea \\ bluefeel0@naver.com, seung.lee@seoultech.ac.kr
}

\begin{abstract}
An important class of features centered on recognition (i.e. the ability to reconize images, speech, gestures, etc) is rapidly becoming available on UX (user eXperience) devices. This requires high performance computing to support recognition with low-latency, low power and high throughput. In this paper, we investigate the UX technologies and find opportunity to accelerate the UX technology on embedded systems.
\end{abstract}

Keywords: User eXperience, Recognition, Hardware Accelerator, MultiProcessor System-on-Chip (MPSoC), Embedded System.

\section{Introduction}

As user interacts more with devices such as game platforms, smart phones, smart $\mathrm{TV}$, entertainment equipments in automobile etc., the enhancing personalized experience became challenging to enable more natural modes of input-output as compared to traditional devices. For instance, various devises that enable selecting or moving object on electronic devices adopting infra-red rays or eye tracking methods without touching devices were introduced. A glove like mouse is already in the market and a 3D air mouse for open space usage is also available, enhancing user experience. Accordingly, game companies are pursuing realistic game interfaces and other conventional companies also are trying to adopt these devices for efficient presentation and education. Clearly, these usage models highlight that the enhancing user experience is gaining significant momentum and such workload need to be analyzed and characterized for embedded systems in terms of performance, costs, and power. In this paper, we first investigate current UX technologies which are promised to bring forth a new spectrum of novel usage models for computing devices in the field of game, entertainment, automotive and medical applications. Then, we discuss an opportunity of accelerating UX technologies for embedded systems by proposing processor architecture based on multi-processor system-on-chip (MPSoC).

The rest of this article is organized as follows. We first describe three different types of motion capture methods and introduce the corresponding UX devices in section 2 . We discuss the opportunity to accelerate the UX technologies on embedded systems and propose the architecture of UX processor adopting application specific hardware accelerators along with CPUs in section 3. Finally, we conclude in section 4 by outlining the direction for future works on this topic.

\footnotetext{
Corresponding Author.
} 


\section{User eXperience (UX) Technologies}

\subsection{Optical Motion Capture Systems for Human Body}

Optical motion capture systems use video cameras to track the motion of markers attached to the particular location of a human body. A passive type of marker uses infrared (IR) LEDs mounted around camera lens. An active type of marker utilizes IR light emitted by the LEDs rather than light reflected from markers avoiding several problems that often plague passive optical systems, including swapping of markers, noisy or missing data and false reflections. The centers of the marker images are extracted from the various camera views using triangulation to compute their frameto-frame positions in 3D space. These systems use a skeleton which is driven by the tracked marker positions as they are captured and the captured skeleton moves around the human's skeleton, which moves the mesh that makes up the skin of the human. Recently, optical motion capturing without using markers are introduced which recognizes gesture and movement of human based on image processing. Fraunhofer's iPointer Presenter enables touch-less operation of a computer through gestures by using two infrared cameras to register a user's finger movements with millimeter precision [1]. At the same time software interprets the recoded data as hand gestures and assigns them to predefined computer commands. Microsoft's Kinect is a controller-free gaming experience for video game platform which enables users to control and interact with the game platform without the need to touch a controller, using gestures and spoken commands. The Kinect is a single sensor bar that contains two depth sensors, and a standard RGB camera. The Kinect also features a built-in microphone that allows voice-activated commands when applicable, and the vocal feature does pop up in games [2].

\subsection{Magnetic Motion Capture Systems for Human Body}

Magnetic motion capture system utilizes sensors placed on the body to measure the low-frequency magnetic field generated by a transmitter source. It is useful to detect hidden objects and provides low cost system compared with the optical motion capture system. However, magnetic motion capture system is not free from electrical wiring due to the shielded cable and is sensitive to the environment in measuring the magnetic field of the objects. A magnetic motion capture system measuring movement of hands and a body simultaneously was introduced [3]. The transmitter for a hand is placed at the same position as the one of a sensor attached on a forearm. A PC for capturing data is connected to two LIBERTYTM systems through USB interfaces, and to the MotionStar Wireless ${ }^{\mathrm{TM}}$ system via the Ethernet cable. They evaluated the system by measuring a folk dance and demonstrated that the proposed system can digitize the hands and the body movement accurately.

\subsection{Mechanical Motion Capture Systems for Human Body}

Mechanical motion capture systems measure the human's joint motion using potentiometer and slider. This method is not affected by typical problems of optical 
and magnetic based systems providing absolute measurement and supports high sampling frequency of motion. However, it has a limitation on natural motion capture due to the attached mechanical devices on the body. Chording glove is a kind of hand input device to measure human-hand postures so that the user can intuitively interact with synthetic objects in a virtual environment in a natural way [4]. The keys of a chord keyboard are mounted on the fingers of a glove. A chord can be made by pressing the fingers against any surface. Shift buttons placed on the index finger enable the glove to enter the full ASCII character set. The chording glove is designed as a text input device for wearable computers and virtual environments. Haptic workstation is a fully integrated simulation system providing right and left wholehand force feedback, immersive $3 \mathrm{D}$ viewing, and easy to use CAD model manipulation [5].

\subsection{Gaze Tracking Systems}

In addition to the whole body capture systems, a gaze tracking system measures point of view or relative movement of eye. With gaze tracking system, users can intuitively interact with application platforms thanks to similar operation protocol with a convention computer mouse. Also, its throughput is very high compared to that of the hand operation based conventional input devices. Heo et al. proposed a realistic game system using a multi-modal interface, including gaze tracking, hand gesture recognition and bio-signal analysis [6]. In order to unify the gaze tracking device and the head mounted display, a near infrared LED illuminator and one camera are attached below the head mounted display.

\section{Accelerating UX Technology on Embedded Systems}

Enhancing user experience has become of significant interest for the usage models such as realist game, mobile devices, wearable computers, and medical instruments etc. to provide natural user interfaces. In order to achieve these usage models, employing heterogeneous motion capture systems together is a suitable approach to provide high-accuracy and to compensate for the limitation of the different motion capture schemes. This heterogeneous UX device is required to keep the following things in key focus: 1) need to ensure that it is user friendly adoptable, 2) need to ensure that it is extremely efficient in terms of low power, low cost and high performance, 3) need to satisfy the application apace (accuracy, execution time, and throughput) and 4) need to provide a general-purpose programming environment for different application developers.

Heterogeneous multi-processor System-on-Chip (MPSoC) is attracted by computing intensive applications due to its capability of drawing strengths of different processors to improve the overall performance. Usually, the computing intensive tasks are mapped to the accelerator processors and the rest of the tasks are mapped to the CPUs in a heterogeneous MPSoC. Researchers have been exploring the integration of domainspecific hardware accelerators in order to improve performance and power efficiency for a class of special purpose applications and application-specific accelerators have been developed for image processing, security, and communication. 
Fig. 1 illustrates the proposed highly efficient UX processor architecture suitable for a large-scale motion capture system based on the heterogeneous MPSoC architecture that integrates low-power CPU cores along with application specific accelerators. In order to provide a low-cost energy efficient architecture, we adopt multiple small cores (e.g. Intel Atom or ARM core). This UX processor consisting of multiple small cores connected through an on-die system interconnect to an integrated memory controller to attach to the main memory. In order to accelerate the UX execution time, we explored the potential of integrating application-specific hardware accelerators for the key primitives of UX devices. All of these accelerators should be key primitives in such motion capture algorithms and be reusable across a wide range of UX workloads for heterogeneous sensors. This heterogeneous architecture is advantageous because it provides significant performance improvement and energy efficiency that is inherent with integrated hardware accelerators as compared to general purpose CPUs.

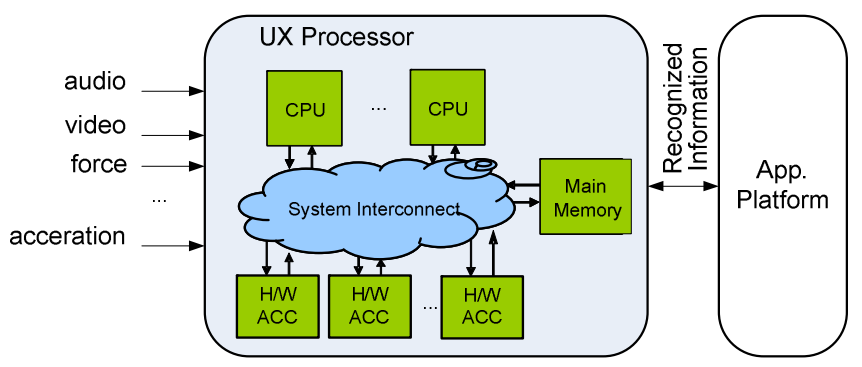

Fig. 1. Architecture of UX processor

\section{Conclusions and Future Works}

In this paper, we investigated motion capture systems for UX devices and presented an opportunity to accelerate the UX technology on embedded systems by proposing the processor architecture based on MPSoC. With emerging UX based applications on game console and smart devices, we plan to continue studying additional workloads and further refining our processor architecture with the right set of accelerators and associated architectural supports. We also plan to simulate the hardware accelerators proposed along with RISC cores in a SoC platform to study the interaction between hardware and software components. We expect that accelerating UX technology on embedded systems will bring forth a new spectrum of novel usage models for entertainment platforms.

\section{References}

1. Fraunhofer, Your Finger as a Remote Control, http://www .hhi . fraunhofer . de/

2. Micorsoft, Introducing Kinect for Xbox 360, http : / /www . xbox. com/kinect/ 
3. Kaiga, T., Yukawa, T., Mitobe, K., Miura, T., Tamamoto, H., Yoshimura, N.: Magnetic motion capture system measuring movements of hands and a body simultaneously. In: ACM SIGGRAPH (2007)

4. Rosenberg, R., Slater, M.: The Chording Glove: A Glove-Based Text Input Device. IEEE T. on Systems, Man, And Cybernetics 29(2) (May 1999)

5. Immersion Corporation, The Haptic Workstation, http: / / www . vrlogic.com

6. Heo, H., Lee, E.C., Park, K.R., Kim, C.J., Whang, M.: A realistic game system using multimodal user interfaces. IEEE Transactions on Consumer Electronics 56(3), 1364-1372 (2010) 